\section{Mercado de Santa Caterina}

Una visión caleidoscópica -y al mismo tiempo híbrida de la realidad-plantea este proyecto en medio de un barrio central en Barcelona: antes que un cuerpo unitario, este edificio construido con múltiples elementos define espacios para un bloque de viviendas, un sitio arqueológico y un mercado. Su fachada principal es la quinta fachada: una cubierta ondulada pensada para verse desde arriba. Palabras clave: Arquitectura - España, rehabilitación urbana, mercado, Barcelona.

A vision of reality both kaleidoscopic and bybrid is offered by this project located in a central area of Barcelona. Rather than a unitary construction, the building made with multiple elements defines spaces for an apartment block, an archeological site and a market. Its main facade is the fifth façade, a wavy roof surface designed to be seen from above. Key words: Architecture - Spain, urban renewal, market, Barcelona.

\section{EMBT arquitectos}

Barcelona, España

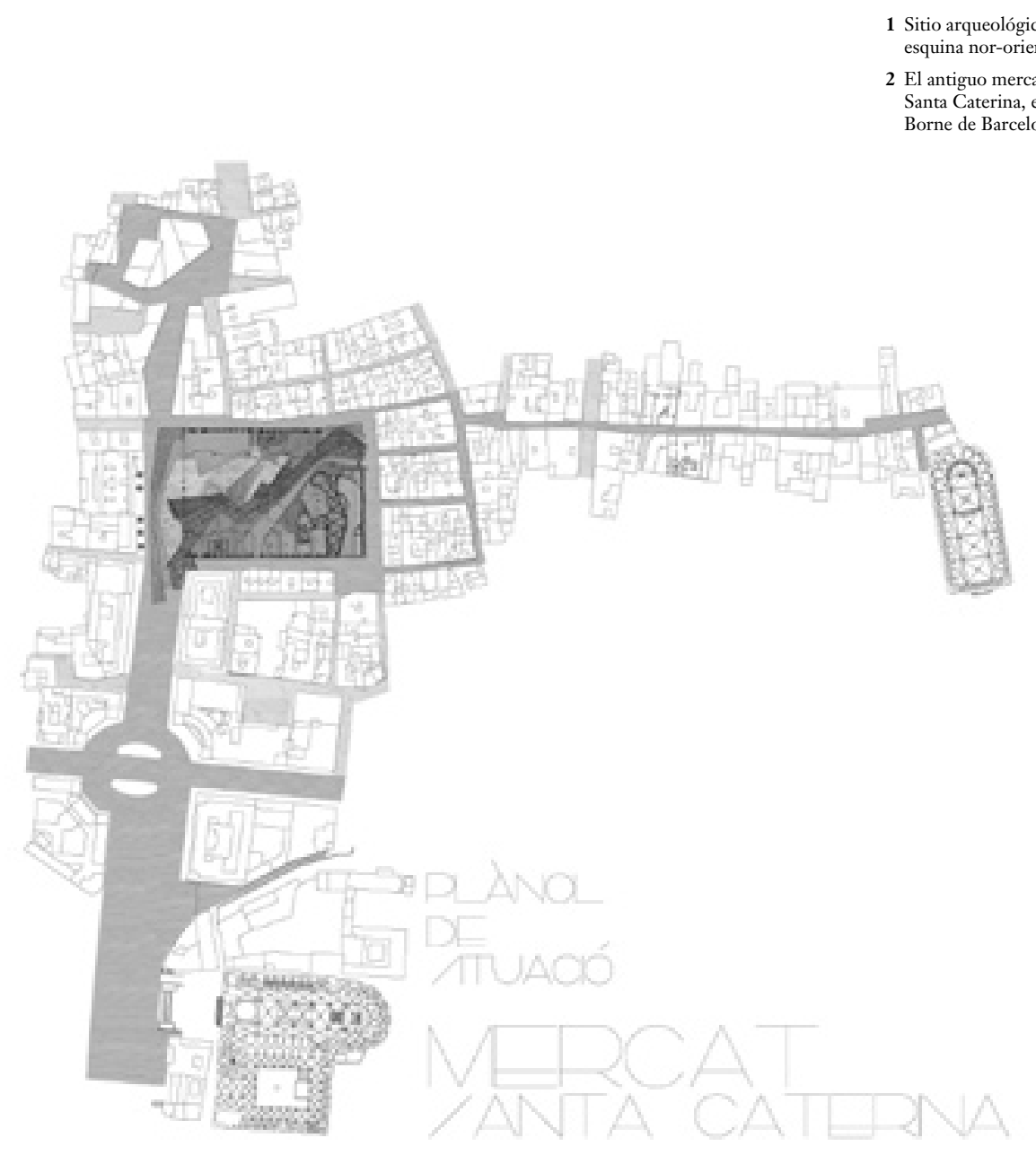

Ciutat Vella a diferencia de otros barrios de Barcelona es una ciudad completa... Quizá ésta sea la más clara cualidad de los centros históricos. A partir de ahí todo se complica. El planeamiento existente no es capaz de gestionar la complejidad de la situación. Y el planeamiento, en su deseo de eficacia inmediata, simplifica hasta límites insoportables las reglas del juego.

I. El primer equívoco es que se pueda hablar de nuevo y viejo. La forma construida tiene una compleja relación con el tiempo. Quizá experimentar en nuestra casa de la calle Mercaders en Ciutat Vella algo parecido a habitar -otra vezlos mismos lugares. Como si habitar no fuera más que moverse en el tiempo de un lugar...

Lo que ha conseguido llegar hasta hoy es actual, útil, contemporáneo. $\mathrm{Y}$ además permite volver hacia atrás en el tiempo para seguir adelante.

II. Otro equívoco es el que defiende el derribo como la única posibilidad de "solucionar" las cosas. Al contrario.

Usar y volver a usar. Es como pensar y repensar las cosas. Y la arquitectura no es más que un modo de pensar sobre realidad.

Así pues las nuevas construcciones se sobreponen a las existentes. Se mezclan, se confunden para hacer aparecer ese lugar en sus mejores cualidades... Así parece lógico usar términos como conglomerado, híbrido, etc. ...Términos que intentan superar la dicotomía del blanco y negro.
III. La superposición de los distintos momentos en el tiempo ofrecen el espectáculo de las posibilidades. Abren un lugar al juego de las variaciones.

Es difícil sacar conclusiones más allá de lo más elemental que define unas mínimas condiciones de vida. Sin embargo, la fuerza de las variaciones constantes sobre un lugar nos colocan en la línea de trabajo. Repetir.

Volver a hacer de nuevo. El proyecto no debe insistir en un momento concreto del tiempo, sino instalarse en él. Nuestro proyecto se inicia con la crítica al planteamiento existente y propone un modelo que permita adaptarse a la complejidad del lugar.

Una normativa que atienda algo más que a la anchura de la calle y la altura de edificación. Un primer esquema que permita desarrollar la complejidad de la ciudad y que respete los compromisos públicos adquiridos.

Proponemos un modelo donde no sea tan fácil distinguir entre rehabilitación y nueva construcción.

Donde las plazas, el trazado continuo de ensanchamientos pasa por encima de la calle como único mecanismo urbano. Se reduce el número de puestos de venta, racionalizando los sistemas de accesos y servicios. Aportando espacio público y densidad residencial. Desplazamos la zona comercial hacia la Avenida Cambó, reduciendo su sección y abriendo la antigua construcción del Mercado hacia el interior del barrio de Santa Caterina. ARQ 

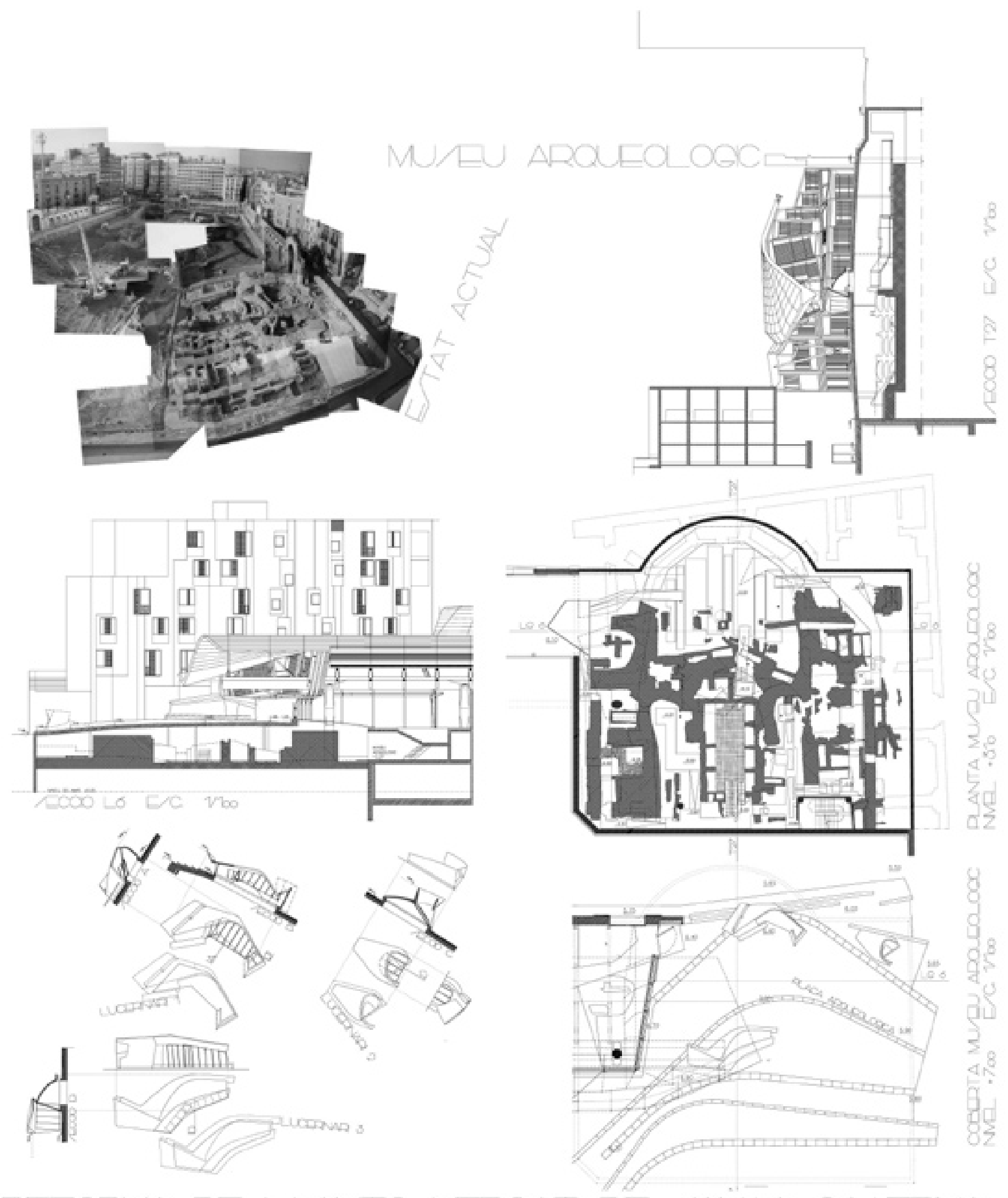

REOPMA DE L'ANTC MERAT DE /ANIA CAI WRNA MEXCAT DE/ANTA CATEANA

RRC MPAIE, DONEDIA TAGLADUE. 
4 Febrero de 2004.

Fotografía de Toni Cumella

5 Plan de composición del

Marzo de 2004

Fotografía de Toni Cumella
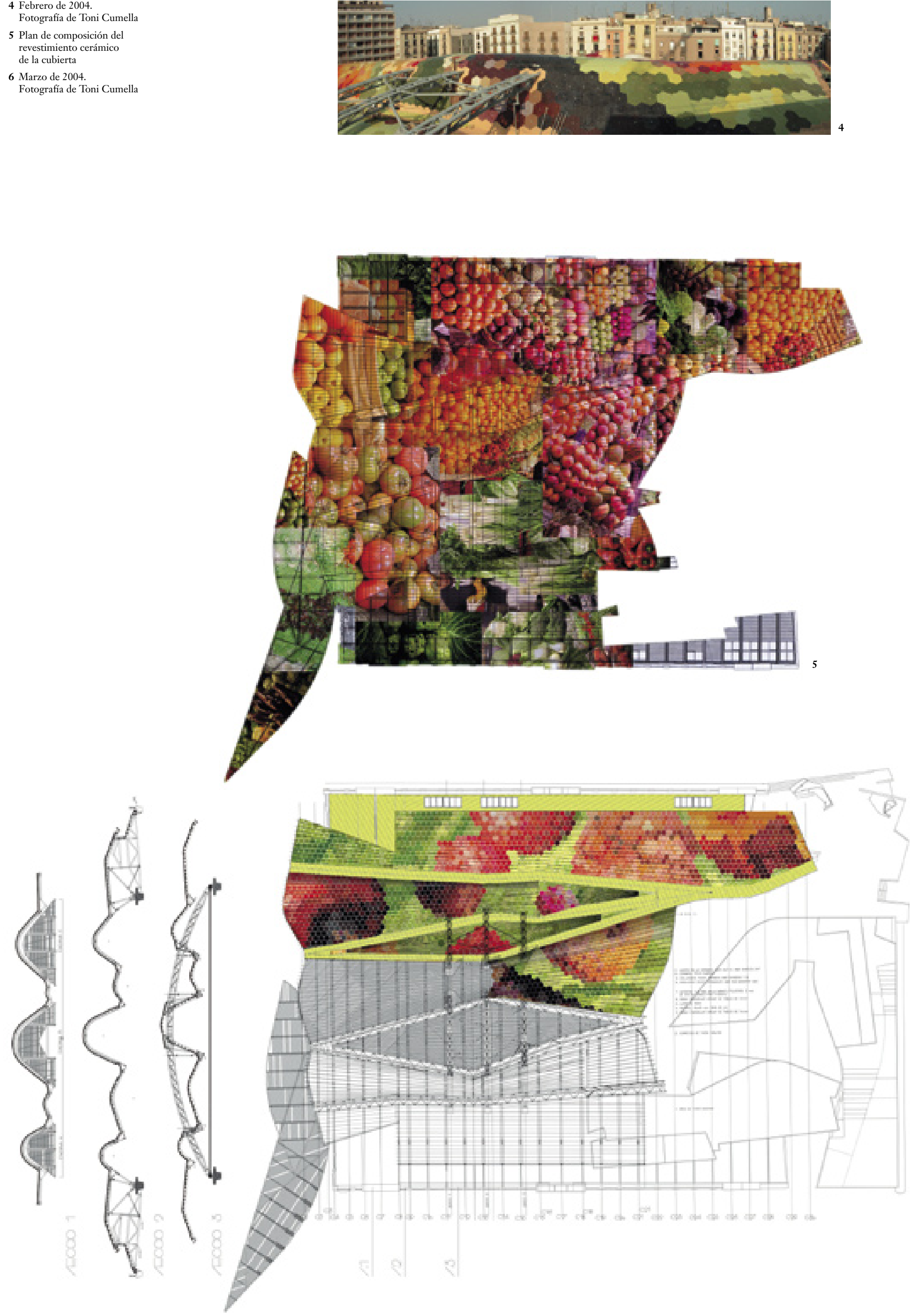

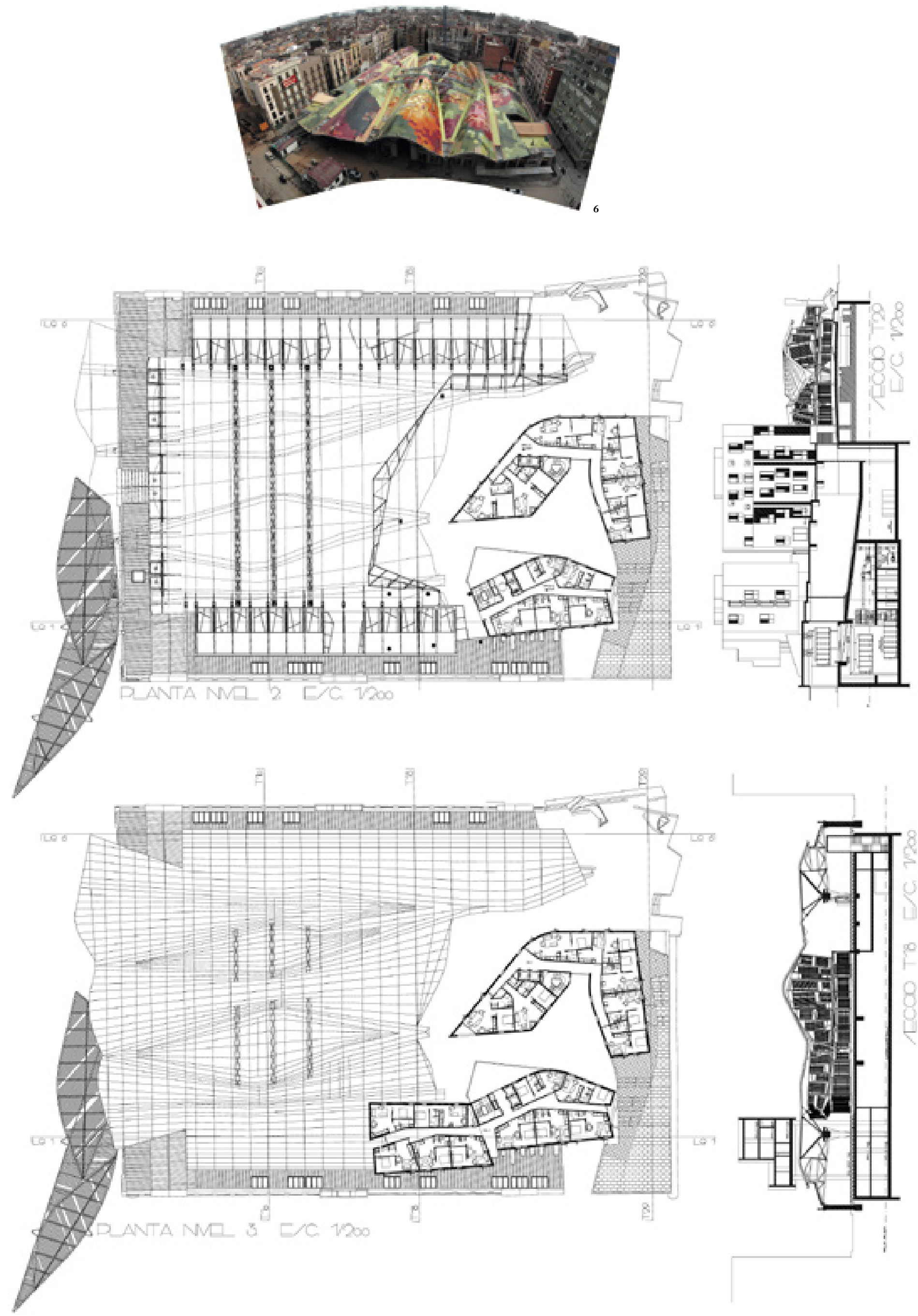

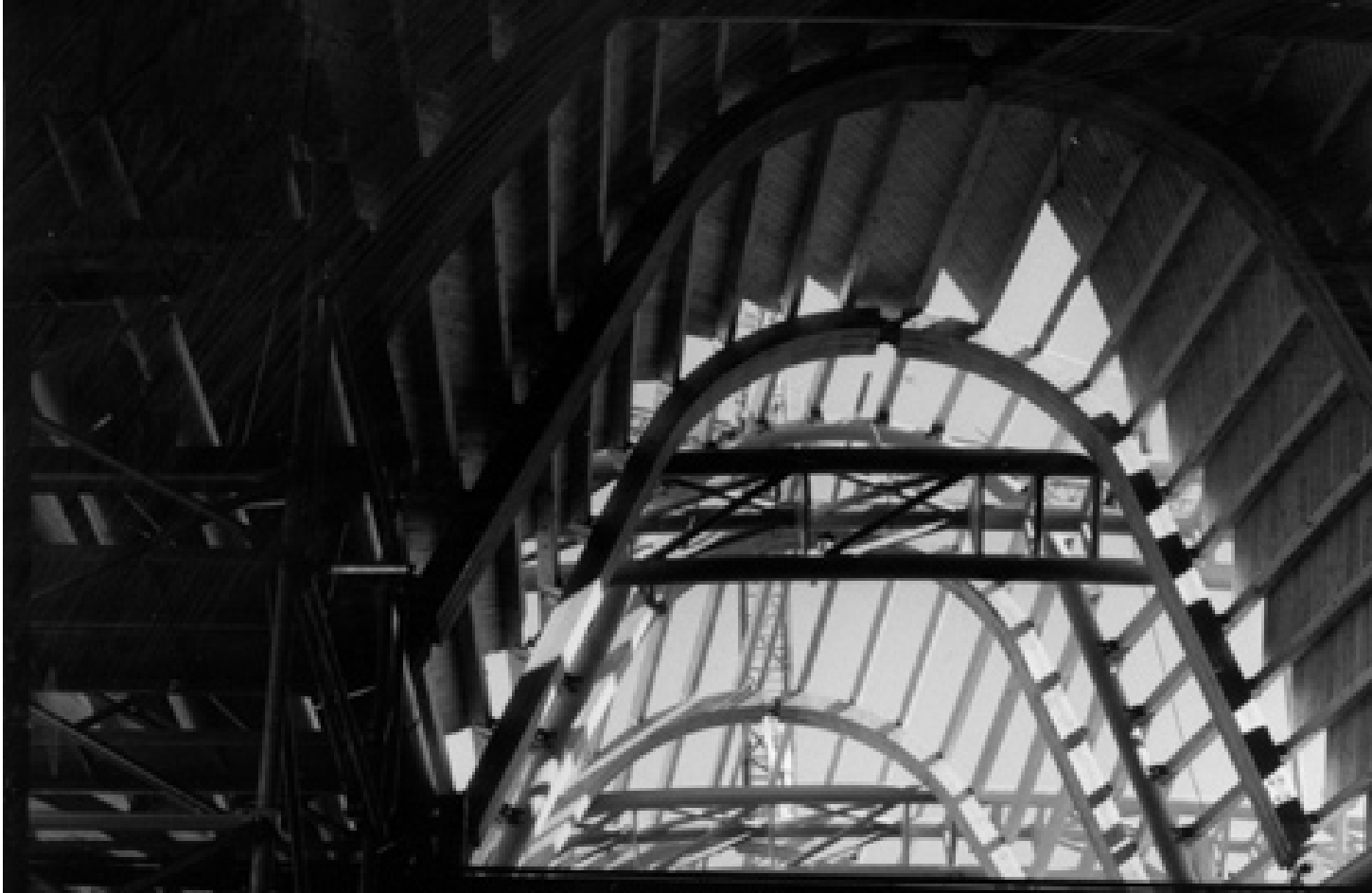

-

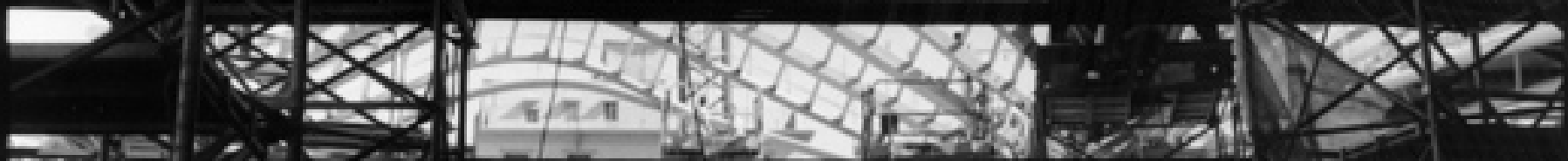

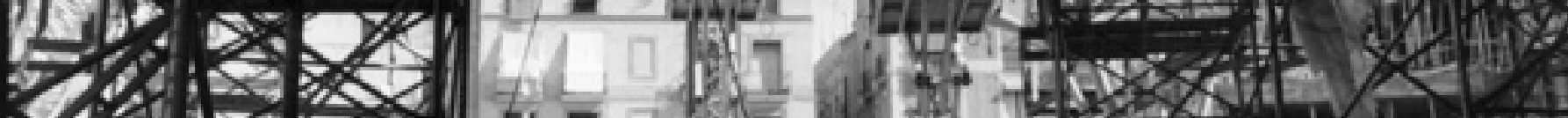

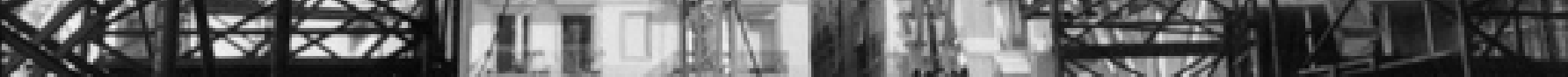
2.

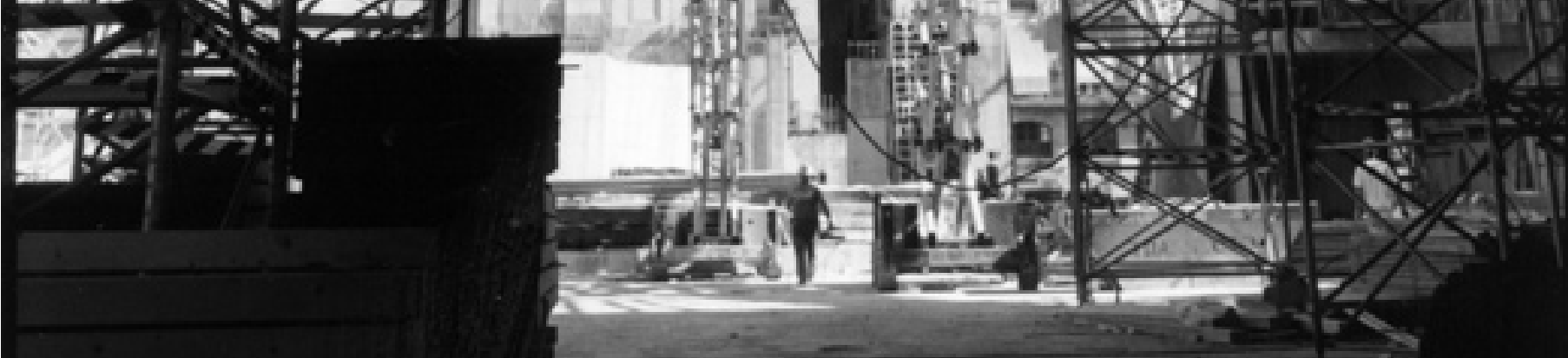



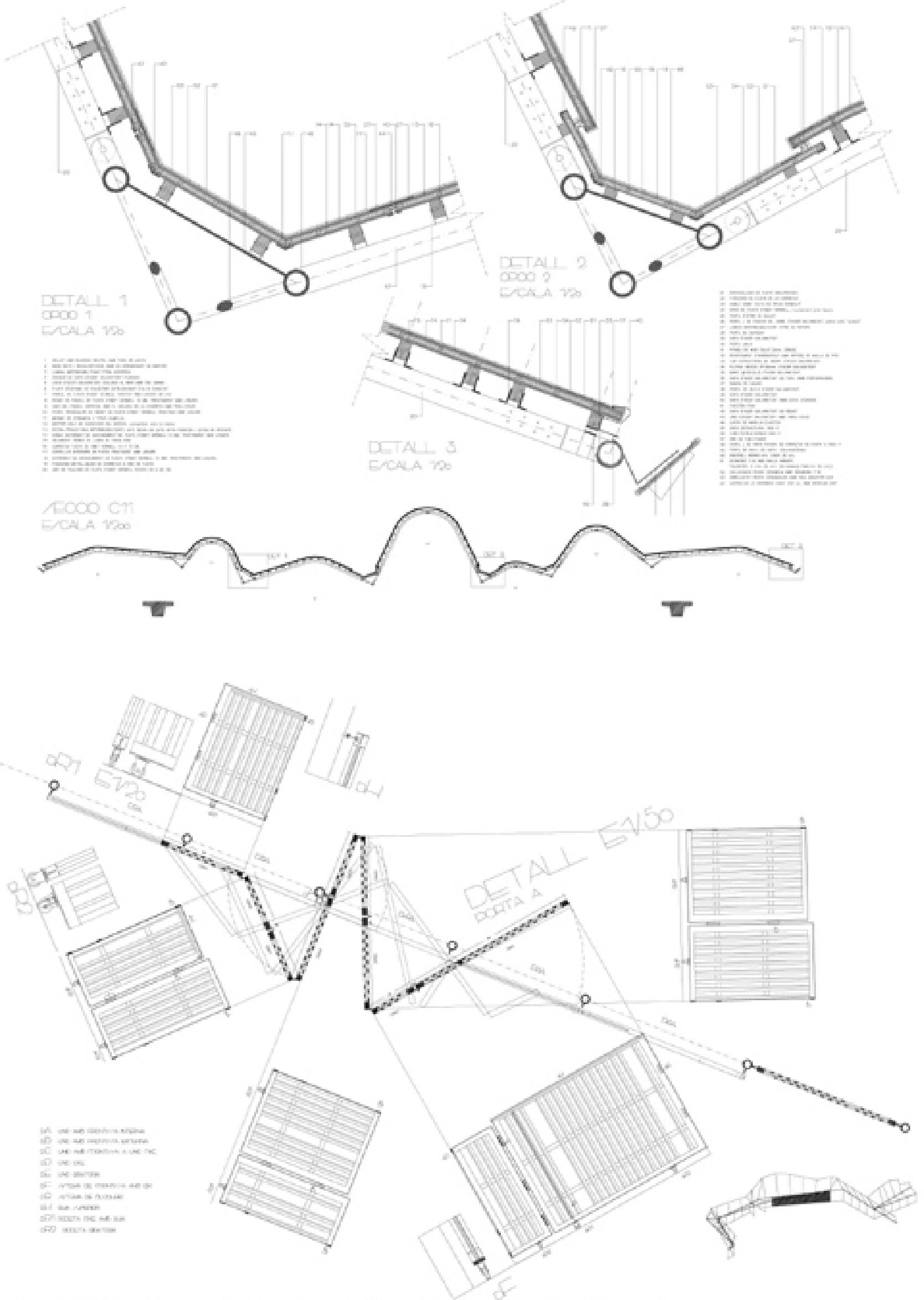


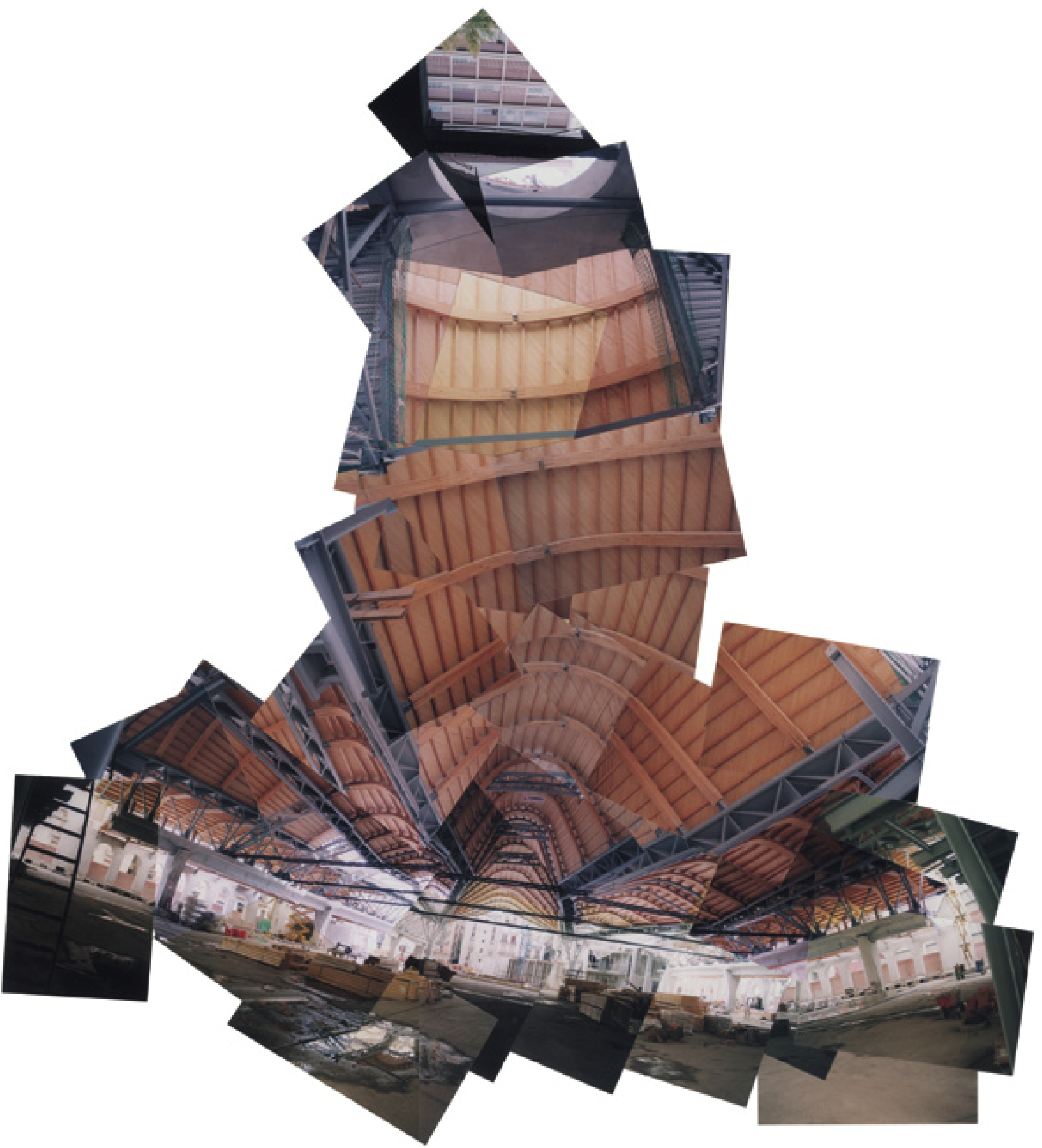




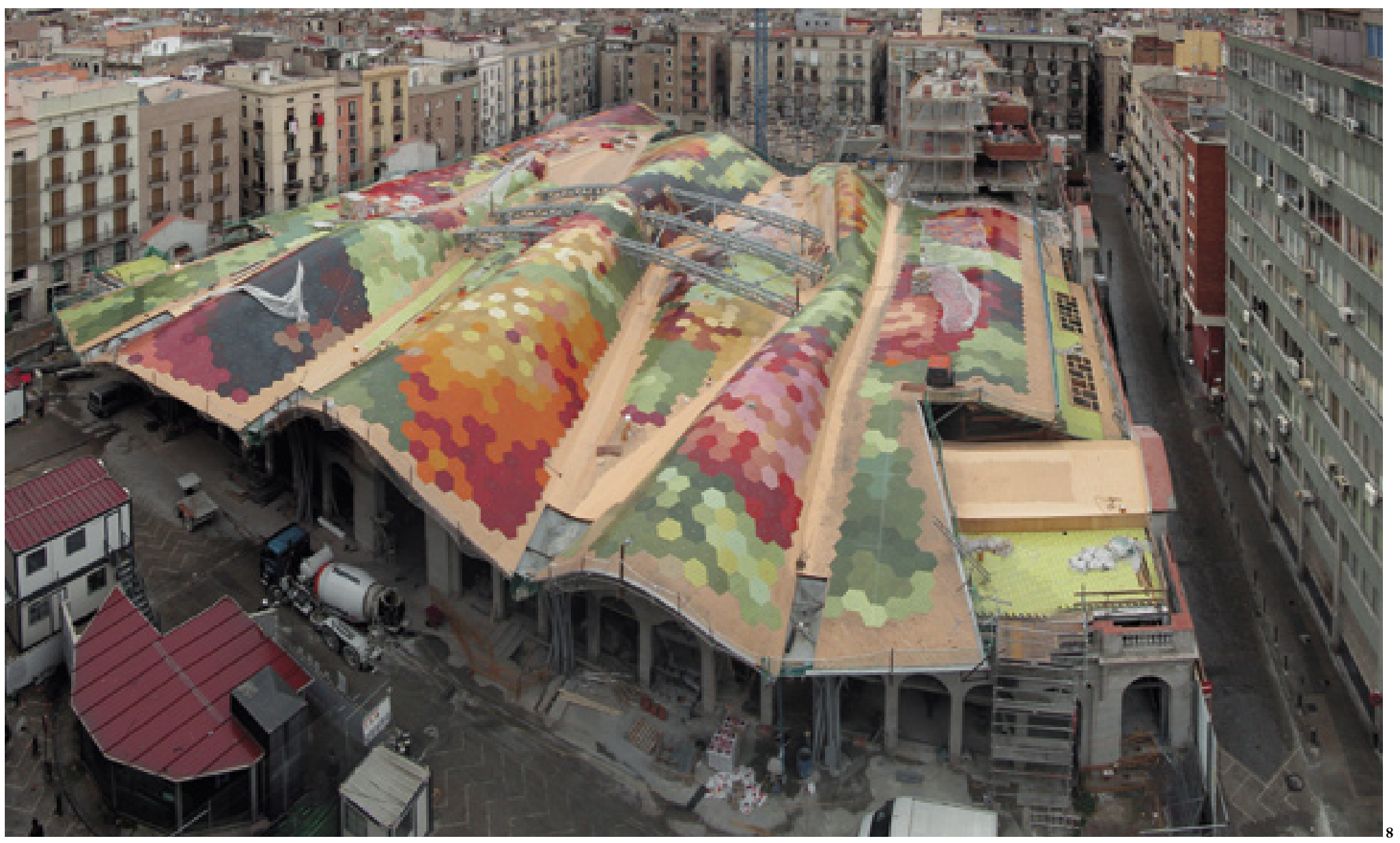

Rehabilitación del Mercado de Santa Caterina Arquitectos: Enric Miralles, Benedetta Tagliabue Jefe de proyecto: Igor Peraza

Colaboradores: Constanza Chara, Hirotaka Koizuni, Josep Miàs, Tomoko Sakamoto, Marta Cases, Joan Poca, Alejandra Vázquez, Marco Darío Chirdel, Josep Belles, Alicia Bramon, Santiago Crespi, Gianfranco Grondona, Laura Valentini, Adelaide Passetti, Lluis Corbella, Jorge Carvajal, Makoto Fukuda,

Andrea Landell de Moura, Isabel Zarasoza, Torsten Skoetz, Adrien Verschuere, Loïc Gestin, Annie Marcela Henao, Ezequiel Cattaneo, Fabián Asunción, Leonardo Giovannozzi, Annette Hoëller, Sabine Bauchmann, Silke Techen Bárbara Oel Brandt, Mette Olsen, Florencia Vetcher, Nils Becker, Montse Galindo, Bárbara Appolloni, Jean François Vaudeville, Peter Sándor Nagy, Massimo Chizzola, Umberto Viotto, Eusenio Cirulli Colaboradores especiales: Ricardo Flores, Eva Prats, Joan Callís Maquetas: Fabián Asunción, Isnacio Quintana, Christian Molina,
Stefan Geenen, Maarten Vermeiren, Torsten Schmid, Tobias Gottschalk, Stefan Eckert, Ute Grolz, Cristine, Thomas Wuttke, Luca Tonella, Stephanie Le Draoullec, Mónica Carrera, Raphael de Montard, Kris Kinder, Luis Felipe, Fernando Alonsez, Nuno Rodrigues, José Latorre, Martina Vigano, Kevin MacPherson, Dirk Mayer, Daniel Erfeld, María Pierres, Annie Kwon, Israel López, Gonzalo Elizarraras, Carolina Civarolo, Valentin Bernard, Desireé Mann, José Fernando Brisaldo, Alexis Bisiaux Ubicación: Avenida Cambó, el Borne, Barcelona, España Cliente: IMMB, Foment de Ciutat Vella

Cálculo estructural: Robert Brufau

Colaboradores: José María Velasco, Miquel Llorens Instalaciones: PGI

Año proyecto: 1997 - 2004

Año construcción: 2003 - 2004

Fotografía: Toni Cumella, Alex Gaultier, EMBT arquitectos 